\title{
Chronic Pyelonephritis Mimicking Renal Mass: A Case Report
}

 \\ 1 University of Health Sciences Turkey, Ankara City Hospital, Clinic of Urology, Ankara, Turkey \\ 2University of Health Sciences Turkey, Ankara City Hospital, Clinic of Pathology, Ankara, Turkey
}

\begin{abstract}
A 72-year-old male patient presented to the hospital with right flank pain, fever and haematuria. Computed tomography revealed a horseshoe kidney, grade 2 hydronephrosis and a focal hyperdense lesion, of which differential diagnosis between mass and abscess could not be made, in the right kidney. Magnetic resonance imaging was performed subsequently and revealed a mass. Right radical nephroureterectomy was then performed. Pathological examination described a fragile, necrotic lesion, which was a sign of chronic pyelonephritis that dilated the pelvicalyceal system. It was also associated with the mucosa and formed cystic structures with a size of $5 \times 4 \mathrm{~cm}$ in the cortex. Chronic pyelonephritis cases with acute episodes should be considered in the differential diagnosis of renal tumours.

Keywords: Chronic pyelonephritis, renal mass, case report
\end{abstract}

\section{Introduction}

Pyelonephritis is defined as the inflammation of the renal parenchyma. Fever, costovertebral angle tenderness, nausea and vomiting might be symptoms of acute illness. Chronic pyelonephritis, which is a nonspecific infection of the kidney, may occur after recurring acute episodes of pyelonephritis (1). Although imaging methods are not routinely used for diagnosis, computed tomography (CT) and magnetic resonance imaging (MRI) can be used to determine hydronephrosis, emphysematous pyelonephritis and abscess in patients with congenital structural anomalies and predisposing factors that increase underlying morbidity, such as diabetes mellitus (2). Horseshoe kidney, which occurs from the fusion of metanephric buds in the $4^{\text {th }}-6^{\text {th }}$ weeks of embryonic development, is the most common renal fusion anomaly, with an incidence of 0.4-1.6 in 10,000 live births. People with a horseshoe kidney are at risk of urogenital system infections, because of malrotation, insufficient drainage and obstruction of the ureteropelvic junction (3). Rarely, imaging findings in atypical clinical presentations may be confusing, mimicking kidney or urothelial carcinomas (2). This case report presents the diagnosis and treatment process of a patient with horseshoe kidney, who presented with acute pyelonephritis that mimics upper urinary tract malignancy in imaging.

\section{Case Presentation}

An informed written consent was obtained from the patient for this case report. A 72-year-old male patient who presented to the emergency department with flank pain of 1-week duration, fever, chills and macroscopic painless haematuria for 3 days was hospitalised. He had no symptoms of dysuria or frequent urination. The patient had recurrent episodes of urinary tract infection, hypertension and coronary artery disease, had undergone radical prostatectomy 6 years ago and had a history of pulmonary embolism after this surgery. Physical examination revealed right costovertebral angle tenderness. His blood pressure, pulse rate and body temperature were $88 / 51 \mathrm{mmHg}$, 89 beats per minute and $38.3{ }^{\circ} \mathrm{C}$, respectively. Laboratory test results revealed white blood cell count of $40 \times 10^{9} / \mathrm{L}$, blood urea concentration of $32 \mathrm{mg} / \mathrm{dL}$ and serum creatinine concentration of $0.92 \mathrm{mg} / \mathrm{dL}$. Complete urinalysis revealed over 204 leucocytes per high power field. Urine culture yielded no growth. Findings of abdominopelvic ultrasonography, performed as the firstline radiological evaluation under emergency conditions, were suboptimal. For this reason, contrast-enhanced abdominal CT was performed, and findings were interpreted as horseshoe kidney with grade 2 hydronephrosis and a focal hyperdense lesion in the upper pole of the right kidney. The relationship of the lesion with the calyces could not be evaluated, and the

Cite this article as: Şenel S, Ceylan C, Ceviz K, Özerdem Y, Gümüşkaya B. Chronic Pyelonephritis Mimicking Renal Mass: A Case Report. Bull Urooncol 2021;20(3):192-195

Address for Correspondence: Samet Şenel, University of Health Sciences Turkey, Ankara City Hospital, Clinic of Urology, Ankara, Turkey Phone: +90 5378802285 E-mail: samet senel_umt@hotmail.com ORCID-ID: orcid.org/0000-0003-2280-4192 Received: 24.10.2020 Accepted: 17.01.2021 
lesion having dense content could not be distinguished as a mass or abscess.

Ertapenem treatment was initiated for pyelonephritis. Although symptoms of pyelonephritis responded to medical treatment, haematuria persisted. MRI was performed, as the differential diagnosis could not be made with contrast-enhanced CT. MRI revealed a mass lesion with a size of $7 \times 5 \times 4 \mathrm{~cm}$, which caused dilatation in the collecting system of the right kidney with extremely thinned parenchyma, extending towards the renal pelvis and showing diffusion restriction (Figure 1A and 1B). The lesion was predicted as renal cell carcinoma (RCC) or transitional cell carcinoma according to the MRI.

Afterwards, the patient was prepared for surgery, and an open right heminephroureterectomy and cuff resection was performed with a pre-diagnosis of a right renal mass. Macroscopic pathological examination was performed on the $11.5 \times 2.2 \mathrm{~cm}$ ureteral material and $17 \times 9.5 \times 6.5 \mathrm{~cm}$ renal material, and a lesion $5 \times 4 \mathrm{~cm}$ in size that completely dilated the pelvicalyceal system, compressed the cortex, with a fragile necrotic appearance, connected to the mucosa and formed cystic structures in the



Figure 1A. Computed tomography image compatible with horseshoe kidney with stones and thinned parenchyma

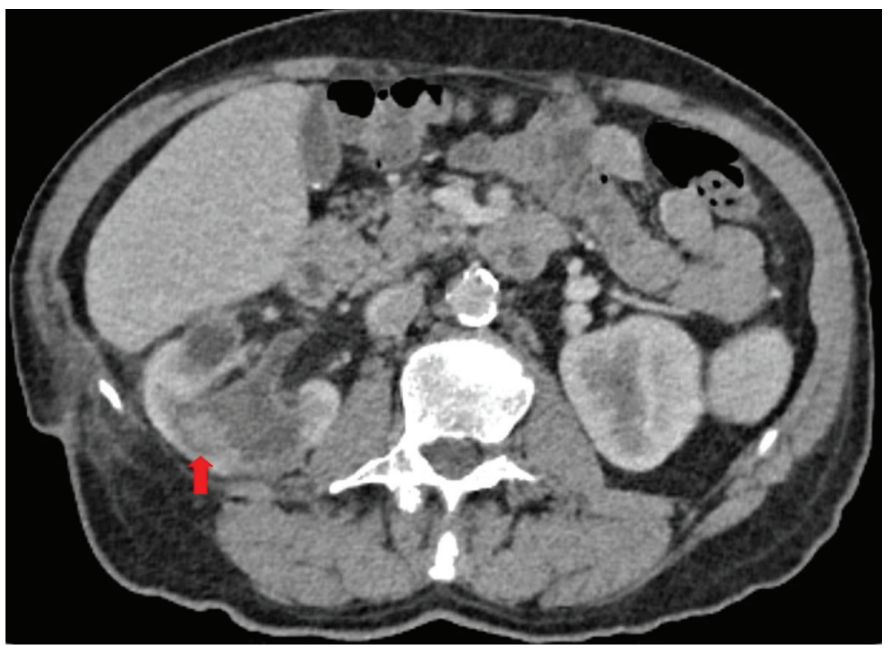

Figure 1B. Hyperdense lesion in the upper pole of the right kidney that cannot be distinguished as mass or abscess cortex, was detected in the renal material sections. Microscopic examination revealed that the described lesion was completely composed of necrosis, fibrin and inflammatory cells without neoplastic changes, and the appearance was compatible with the findings of chronic pyelonephritis showing active chronic inflammation and necrosis (Figure 2A and 2B). The patient was discharged on postoperative day 5 and did not have any problems in the follow-up.

\section{Discussion}

Chronic pyelonephritis is an inflammation of the parenchyma and pyelocaliceal system of the kidney that occurs as a result of recurrent acute episodes over the years. Chronic pyelonephritis is a nonspecific diagnosis made as a result of excluding specific chronic variants of kidney infections, such as xanthogranulomatous and emphysematous pyelonephritis. Generally, the diagnosis is made when end-stage renal failure develops (4). A study showed that the primary cause of end-stage renal disease is chronic pyelonephritis in $13 \%$ of the patients. It occurs in 1-2 per 1.000 women and in 0.5 per 1.000 men (5). Obstructive uropathy, untreated urogenital system infections,

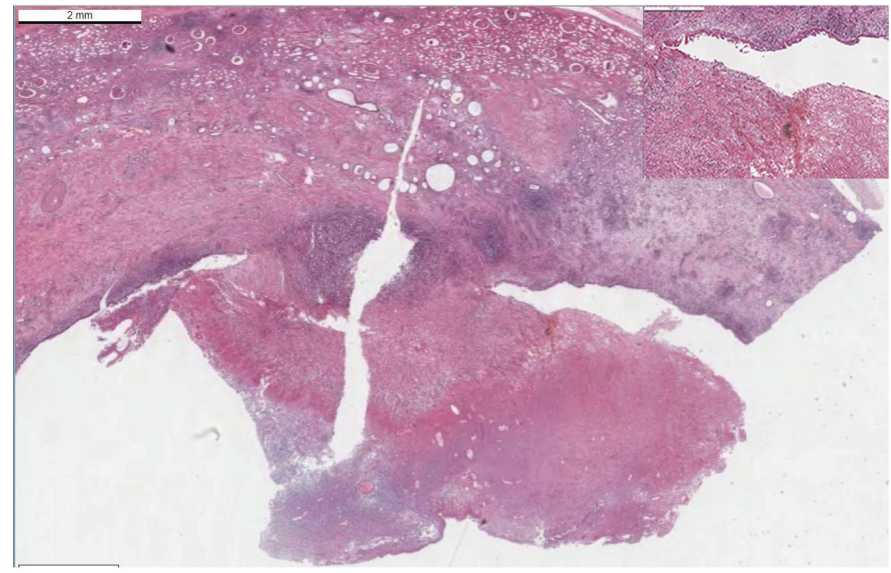

Figure 2A. Necrotic mass lesion, which forms a polypoid mass macroscopically, in the background of chronic pyelonephritis



Figure 2B. Inflammatory cells and tubular atrophy thyroidisation 
schistosomiasis and vesicoureteral reflux are risk factors for chronic pyelonephritis. Although the incidence of RCC does not change in patients with horseshoe kidney, urinary stones and recurring urinary system infections are more common due to the obstruction caused by the abnormal anatomy of the kidney (3). In the present case, the patient had multiple stones, grade 2 dilatation and pyuria in the right kidney.

The clinical presentation of chronic pyelonephritis is related to the disease course. Similar to the present case, findings such as fever and flank pain may occur during acute episodes. However, clinical and urinalysis findings are not correlated. Bacteriuria and pyuria, which are the differential findings of urinary tract infection during acute episodes, are not predictive. The urine may be sterile in cases where the ureter or calyx is completely obstructed (6).

Radiological evaluation is required in complicated pyelonephritis in the presence of accompanying conditions such as renal colic, kidney stones, diabetes, infection with a virulent organism, history of previous surgery and immunosuppression, a history of recurrent pyelonephritis attacks and sepsis (7). For this purpose, CT, which is used as a first-line imaging method for diagnosis, can be used in low-dose renal colic protocol as well as in the portal phase (2). CT findings of chronic pyelonephritis are characterised by parenchymal atrophy of the affected areas, hypertrophy of residual normal parenchymal tissue, clubbing of the calyces secondary to retraction of the papilla from adjacent overlying renal scarring, dilatation of the calyces and overall renal asymmetry. Contrasted CT is important to differentiate the non-enhancing areas of infarction from the scar tissue; it can also help in the differential diagnosis of pseudotumors due to focal parenchymal hypertrophy from the renal neoplasm (8). If renal findings cannot be fully characterised by $C T$, advanced techniques can be used. With MRI, which has a better soft tissue resolution, anatomical boundaries can be distinguished better, disease spread can be evaluated further and infiltrative infectious formations that mimic pathologies such as urothelial carcinoma, lymphoma and metastatic disease can be distinguished (9). However, despite these imaging methods, certain benign pathological formations cannot be distinguished from malignant lesions of the kidney. These lesions, called renal pseudotumors, are caused by developmental, granulomatous and vascular pathologies as well as inflammatory/infectious conditions including chronic pyelonephritis (10). Renal inflammatory pseudotumors are rarely localised in the kidney $(11,12,13,14,15,16,17)$. Although renal inflammatory pseudotumors are rare lesions, they are one of the differential diagnoses of renal malignancy and thus should be considered to avoid misdiagnosis and prevent inappropriate resections (18). In the present case, mass-abscess distinction of the lesion causing dilatation in the right kidney could not be made on CT images. Therefore, MRI evaluated the lesion as a malignancy (RCC or urothelial cancer), indicating that it showed diffusion restriction and enhancement.

The treatment of choice for chronic pyelonephritis varies, as it is a nonspecific diagnosis. Medical and surgical management of the complications are the main goal of treatment. Acute episodes are generally treated with antibiotics and drainage, if necessary. However, in the presence of an atrophic kidney or underlying conditions causing uncontrollable hypertension or recurrent infections, nephrectomy must be considered an option (6). Diagnosis is confirmed by histopathological examination of the nephrectomy material. Microscopic findings of chronic pyelonephritis are nonspecific and presented as tubulointerstitial mononuclear inflammation of the cortex and medulla. The glomeruli are also surrounded by inflammation, but the tubulointerstitial compartment is severely affected. Severe tubular atrophy may end with a finding called 'thyroidisation', which is not a specific finding for chronic pyelonephritis and can be seen in all conditions leading to tubular atrophy. Lymphocytic inflammation is encountered in the submucosa of the renal pelvis (19).

Xanthogranulomatous pyelonephritis is a chronic granulomatous inflammation that destroys the renal parenchyma. CT is the most useful examination in the diagnosis of xanthogranulomatous pyelonephritis. CT revealed a large reniform mass with the renal pelvis tightly surrounding the central calcification without pelvic dilatation. It is commonly indistinguishable from RCC on ultrasound or CT images, and radical nephrectomy is a viable option for treatment when malignancy cannot be ruled out (6). However, cases in which chronic pyelonephritis, a nonspecific infection, cannot be distinguished from renal mass are rare, and the appropriate treatment method is open to discussion. In the present case, although the patient presented with acute pyelonephritis, the structure of the horseshoe kidney, hydronephrotic appearance and thinned parenchyma of the right kidney suggested chronic pyelonephritis.

In the European Association of Urology guidelines, renal biopsy is recommended if radiological findings are suspicious for malignancy (20). However, in the present case, considering the structure of the right kidney, in which a mass lesion was described on imaging and the fact that it was a source of infection, radical nephro-ureterectomy and cuff resection were performed on the patient, as biopsy was not necessary.

In conclusion, chronic pyelonephritis cases with clinical acute episodes in patients with renal anomalies may give present as a renal mass radiologically. Differential diagnosis must be made to prevent unnecessary treatment. In the differential diagnosis of renal tumours, cases of chronic pyelonephritis developed in the background of chronic obstruction should also be considered.

\section{Acknowledgements}

Publication: The results of the study were not published in full or in part in form of abstracts.

Contribution: There is not any contributors who may not be listed as authors.

Conflict of Interest: No conflict of interest was declared by the authors.

Financial Disclosure: The authors declared that this study received no financial support.

\section{Ethics}

Informed Consent: An informed written consent was obtained from the patient for this case report.

Peer-review: Externally peer-reviewed. 


\section{Authorship Contributions}

Concept: S.Ş., Y.Ö., B.G., Design: S.Ş., K.C., Data Collection or Processing: K.C., Analysis or Interpretation: Y.Ö., Literature Search: C.C., K.C., Writing: S.Ş.

\section{References}

1. Huang JJ, Tseng CC. Emphysematous pyelonephritis: Clinicoradiological classification, management, prognosis, and pathogenesis. Arch Intern Med 2000;160:797-805.

2. Udare A, Abreu-Gomez J, Krishna S, et al. Imaging of renal infections that mimic malignancy. Can Assoc Radiol J 2019;70:424-433.

3. Fernandez A, Sherer B, Stoller ML. Laparoscopic heminephrectomy of chronically obstructed horseshoe kidney moiety with staghorn calculus, massive pyonephrosis, and xanthogranulomatous pyelonephritis. J Endourol Case Rep 2018;4:39-41.

4. Ademola BL, Atanda AT, Aji SA, Abdu A. Clinical, morphologic and histological features of chronic pyelonephritis: An 8-year review. Niger Postgrad Med J 2020;27:37-41.

5. Lager DJ, Abrahams N. Practical Renal Pathology, A Diagnostic Approach, 1st ed. A volume in the pattern recognition series: 139. Philadelphia: Elsevier Saunders; 2013.

6. Moss BF, Potter L, Cliff A, Kumar M. Xanthogranulomatous pyelonephritis with associated renal cell carcinoma. BMJ Case Rep 2019;12:e232097. doi: 10.1136/bcr-2019-232097.

7. Song SK, Song JS, Hwang HP, Lee S. Acute pyelonephritis including an overlooked renal cell carcinoma. Clin Nephrol Case Stud 2013;1:7-9.

8. El-Ghar MA, Farg H, Sharaf DE, El-Diasty T. CT and MRI in urinary tract Infections: a spectrum of different Imaging findings. Medicina (Kaunas) 2021;57:e32. doi: 10.1136/bcr-2019-232097.
9. Laissy JP, Idée JM, Fernandez P, et al. Magnetic resonance imaging in acute and chronic kidney diseases: present status. Nephron Clin Pract 2006;103:50-57.

10. Bhatt S, MacLennan G, Dogra V. Renal pseudotumors. AJR Am J Roentgenol 2007;188:1380-1387.

11. Kawamura E, Habu D, Tsushima $H$, et al. A case of hepatic inflammatory pseudotumor identified by FDG-PET. Ann Nucl Med 2006;20:321-323.

12. Schoder H, Larson SM. Positron emission tomography for prostate, bladder, and renal cancer. Semin Nucl Med 2004;34:274-292.

13. Sulu E, Damadoglu E, Berk Takir $H$, et al. A case of endobronchial inflammatory pseudotumor invading the mediastinum. Tuberk Toraks 2011;59:77-80.

14. Sato M, Takasaka I, Okumura T, et al. F-18 fluorodeoxyglucose accumulation in an inflammatory pseudotumor of the spleen. Ann Nucl Med 2007;21:521-524.

15. Huellner MW, Schwizer B, Burger I, et al. Inflammatory pseudotumor of the lung with high FDG uptake. Clin Nucl Med 2010;35:722-723.

16. Gohy S, Hubert C, Deprez P, et al. Benign biliary inflammatory pseudotumor mimicking a Klatskin tumor. Hepatogastroenterology 2007; 54:1348-1352.

17. Alongi F, Bolognesi A, Gajate AM, et al. Inflammatory pseudotumor of mediastinum treated with tomotherapy and monitored with FDGPET/CT: case report and literature review. Tumori 2010;96:322-326.

18. Lee JH, Lee KG, Park HK. Inflammatory pseudotumor of the kidney mimicking malignancy on 18F-FDG PET/CT in a patient with diabetes and hepatocellular carcinoma. Clin Nucl Med 2012;37:699-701.

19. Hou J, Herlitz LC. Renal Infections. Surg Pathol Clin 2014;7:389-408.

20. Bonkat G, Bartoletti R, Bruyere F, et al. EAU Guidelines on Urological Infections. Edn. presented at the EAU Annual Congress Amsterdam, 2020. Available from: uroweb.org/guideline/urological-infections/ 